\title{
The Stressful Implications of Remote E-Working: Evidence from Europe
}

\author{
Ylenia Curzi ${ }^{1}$, Tommaso Fabbri ${ }^{1} \&$ Barbara Pistoresi ${ }^{1,2}$ \\ ${ }^{1}$ Department of Economics "Marco Biagi”, University of Modena and Reggio Emilia, Modena, Italy \\ ${ }^{2}$ RECent, Center for Economic Research, University of Modena and Reggio Emilia, Modena, Italy \\ Correspondence: Department of Economics "Marco Biagi”, University of Modena and Reggio Emilia, Viale \\ Berengario 51, 41121, Modena, Italy. E-mail: ylenia.curzi@unimore.it
}

Received: April 28, 2020

doi:10.5539/ijbm.v15n7p108
Accepted: May 20, 2020

Online Published: June 8, 2020

\begin{abstract}
This paper investigates the importance of different modes of spatial flexibility as well as of the distinction between autonomy and discretion to find plausible explanations of the autonomy paradox, which maintains that the more the job autonomy that remote e-workers have the greater the effort they put into their work with adverse effects on work-related stress. Using multiple regressions, we test the hypotheses regarding the direct influence of autonomy, discretion and work intensification as well as their interaction effects on occupational stress in two subsamples of 1.380 home-based e-workers and 2.574 mobile ones drawn from the 2015 European Working Conditions Survey. The main findings are as follows. Home-based e-workers perceive that autonomy (namely over work goals) directly decreases occupational stress and buffers work intensification (i.e. autonomy over work goals and in the organizational choices of their department/company). In the context of remote e-work, discretion is more likely to boost the stressful impact of work intensification when work is mobile. At the same time, we do not find that autonomy increases work intensification, neither among mobile e-workers, nor among home-based e-workers (for whom it buffers the adverse impact of work intensification). In summary, this study does not confirm the existence of an autonomy paradox associated with remote e-work. Contrarily, it suggests that such a paradox is more likely to surface when research relies on conceptual frameworks that ambiguously define autonomy in terms of what should be more properly conceptualized as discretion.
\end{abstract}

Keywords: remote e-working, autonomy, discretion, work intensification, work related stress

\section{Introduction}

Fostered by the new opportunities enabled by digitalization, remote e-work is becoming an increasingly popular work mode in many developed countries (Charalampous, Grant, Tramontano, \& Michailidis, 2018; European Foundation for the Improvement of Living and Working Conditions [EUROFOUND] and the International Labour Office [ILO], 2017), enabling workers to choose where, when and how to perform their daily work activities (Gajendran \& Harrison, 2007; Gajendran, Harrison, \& Delaney-Klinger, 2015).

Research however suggests that this may have paradoxical consequences for individual wellbeing: the more the job autonomy that remote e-workers have the greater the effort they put into their work with adverse effects on individual wellbeing (Cavazotte, Lemos, \& Villadsen, 2014; Mazmanian, Orlikowski, \& Yates, 2013; Kelliher \& Anderson, 2010; Putnam, Myers, \& Gailliard, 2014). One possible explanation for these contradictory findings is that extant studies do not distinguish between different groups of remote e-workers (Neirotti, Paolucci, \& Raguseo, 2013), neglecting that perception of working conditions and consequences of remote e-work on individual wellbeing may differ depending on the kind of remote location (i.e. the mode of spatial flexibility: home vs. customers' premises, the field or public spaces) (Charalampous et al., 2018). Another possible explanation is that extant research relies on an ambiguous definition of autonomy, using it and discretion as interchangeable terms. Exceptions are Albano, Curzi, Parisi and Tirabeni (2018) who show that a robust analytic distinction between autonomy and discretion (Maggi, 2016) provide a more nuanced understanding of the likely changes in the regulation of work at the micro level of analysis associated with remote e-work.

The aim of this paper is to contribute to this debate, exploring the importance of distinguishing between different modes of spatial flexibility as well as between autonomy and discretion as a way to find plausible explanations of the so-called autonomy paradox. Specifically, it explores whether the direct relationships of autonomy, 
discretion and work intensification as well as their interaction effects on individual wellbeing vary depending on the "where" remote e-work is undertaken.

Moreover, like previous studies (e.g. Suh \& Lee, 2017), we test the hypotheses drawn from literature and regarding the direct influence of autonomy, discretion and work intensification as well as their interaction effects on the wellbeing of remote e-workers in general, separately on two subsamples of 1.380 home-based e-workers and 2.574 mobile ones drawn from the 2015 European Working Conditions Survey (European Foundation for the Improvement of Living and Working Conditions [EUROFOUND], 2017).

The article is structured as follows. The first section presents the theoretical framework and research hypotheses. The next two sections are respectively devoted to the research methodology and results. The paper concludes with a discussion of the main theoretical, empirical and practical implications.

\section{Literature Review and Hypotheses}

Several studies on the effects of remote e-work focus on occupational stress as primary wellbeing dimension (Allen, Golden, \& Shockley, 2015). They also adopt the job demand-control (JD-C) (Karasek, 1979) and the job demands-resources (JD-R) (Demerouti, Bakker, Nachreiner, \& Schaufeli, 2001) models. Consistently, they stress the importance of job autonomy, which is a key feature of remote e-work, as a valuable resource possibly reducing occupational stress (Gajendran et al., 2015; ter Hoeven \& van Zoonen, 2015; Sardeshmukh, Sharma, \& Golden, 2012). These studies provide an ambiguous definition of autonomy in terms of "the possibilities for the individual to choose where, when and how to perform his/her work". Later studies question such an approach. Based on a robust analytical distinction between autonomy and discretion (cf. Maggi, 2016), Albano et al. (2018) show that "the possibilities for the individual to choose where, when and how to perform his/her work" should be more appropriately defined in terms of discretion rather than of autonomy. While autonomy refers to the capability of an individual or collective subject to produce his own rules and manage his own processes of action and decision, discretion indicates room for maneuver in a pre-regulated process, where the subject is required to make decisions choosing from pre-determined alternative actions and decisions (Maggi, 2016). Albano et al. (2018) demonstrate the importance of the above distinction to provide a more nuanced picture of the implications of remote e-work on the regulation of work at the micro-level of analysis, suggesting that that distinction may also help to unpack the consequences of remote e-work on individual wellbeing. Consistent with prior research, this study finds that remote e-workers have greater discretion over their work schedule, work pace, and work methods compared to traditional ones. Scholars argue that this may drive positive effects on occupational stress (Gajendran \& Harrison, 2007; Van Steenbergen, van der Ven, Peeters, \& Taris, 2018).

Remote e-workers are also more likely to experience autonomy (specifically over the choices regarding work goals, colleagues, and the organization of one's department or company) (Albano et al., 2018). However, due to the overemphasis of extant research on work discretion, so far the role of autonomy for remote e-workers' occupational stress has been largely unexplored. Among the few exceptions, there are Konradt, Hertel and Schmook (2003) who show that autonomy over work goals may have beneficial effects.

Scholars also consider involvement in autonomous work groups (Golden \& Veiga, 2005). It has been suggested that this may have beneficial effects even in the context of remote e-working because latest advances in information technologies allow team members to communicate effectively over spatial and temporal boundaries (ter Hoeven \& van Zoonen, 2015).

Based on the above arguments, we test the following:

H1: In the remote e-workers' perception, discretion and autonomy negatively relate to occupational stress.

On the other hand, several studies highlight that work intensification, driven by frequent work interruptions, long working hours, lack of recovery time, and the demand to work during one's free time, at high speed and to tight deadlines, and to set one's pace of work according to a greater number of external determinants, is another key characteristic of remote e-working, possibly inducing occupational stress (Felstead \& Henseke, 2017; Suh \& Lee, 2017; Cavazotte et al., 2014; Grant, Wallace, \& Spurgeon, 2013, Mazmanian et al., 2013; Vendramin, 2007). Accordingly, we hypothesize that:

$\mathrm{H} 2$ : In the remote e-workers' perception, work intensification positively relates to occupational stress.

Research based on the JD-C model argues that the stressful impact of work intensification may be offset by the possibilities for remote e-workers to choose when, where and how to work. Contrarily, extensive qualitative research (Cavazotte et al., 2014; Mazmanian et al., 2013; Azad, Salamoun, Greenhill, \& Wood-Harper, 2016; Kelliher \& Anderson, 2010) and a couple of quantitative studies (e.g. Gajendran et al., 2015; Biron \& van Veldhoven, 2016) show that such possibilities boost the stressful impact of work intensification generating a 
so-called autonomy paradox (Putnam et al., 2014). The above counterintuitive effect is also more likely when individuals work remotely rather than in the standard workplace.

While an extensive body of research documenting an autonomy paradox associated with remote e-work by now exists, potential explanations for this are still limited and this calls for research that engages more thoroughly with the above paradox (Boell, Cecez-Kecmanovic, \& Campbell, 2016). The literature suggests that one way to do this is to distinguish between different types of remote e-workers based on the location from where they work (Neirotti et al., 2013). Yet, to the best of our knowledge, the study of Garett and Danziger (2007) is the only attempt to test this hypothesis. This research reveals that perception of working conditions and occupational stress varies depending on the work location; it also shows that the type of remote e-work location accounts for the likelihood of perceiving that the more autonomy employees have the more difficulties they experience in keeping up with their workload. This study however fails to clarify why certain groups of remote e-workers (i.e., those working at home and those working at home and in the field) experience higher job pressure even though they enjoy higher job autonomy.

A possible explanation of this is that the above study, like all remote e-work research that addresses the autonomy paradox rely on an ambiguous definition which confuses autonomy with discretion.

This suggests that one way to deal with the autonomy paradox is not only to distinguish between different types of remote e-workers based on their work location (i.e., home-based and mobile e-workers) but also between autonomy and discretion.

Accordingly, unlike the mainstream approach and in accordance with Albano et al. (2018), we refer to "the possibilities for remote e-workers to choose where, when and how to work" as discretion. In addition, in contrast to the JD-C and JD-R models and in line with Thompson (1967), we assume that discretion is primarily an organizational requirement: the greater the uncertainty the organization has to cope with, the greater the organization's need that organizational members exercise discretion. However, not necessarily individuals are motivated to exercise discretion. On the other hand, "complex organizations often encourage individuals to exercise organizational discretion at considerable personal sacrifice" (Thompson, 1967/2003, p. 121). In summary, Thompson's conceptual framework underlines that, far from being a paradox, the exercise of discretion may negatively affect individual wellbeing. In the context of remote e-work, this may occur because discretion boosts the stressful implications of work intensification.

We expect that mobile e-workers are more likely than home-based ones to perceive that discretion acts as a booster rather than as a buffer of the relationships between work intensification and occupational stress. Extant qualitative research indeed suggests that this group of workers have to manage relationships with a higher number of interest groups (besides bosses and colleagues, multiple different clients) (Vartiainen \& Hyrkkänen, 2010). This, combined with the ease of connectivity enabled by new digital technologies that rises others' implicit expectations that the individual will be constantly available, may increase the likelihood that mobile e-workers will use their discretion to respond quickly to incoming requests from a greater number of different interest groups, ending up working everywhere/all the time.

Regarding autonomy, to the best of our knowledge, no empirical effort has been made to date to engage with its potential moderating role in the relationships between work intensification and remote e-workers' perceived occupational stress. Therefore, in an attempt to provide empirical evidence on this issue, this study explores the potential moderating effect of autonomy and if it changes depending on the location of remote e-work.

Considering all the aforementioned arguments, we test the following:

H 3a: Discretion and autonomy moderate the association between work intensification and occupational stress as perceived by remote e-workers.

H3b: Such a moderating role differs depending on whether e-work is performed at home or in not-fixed, remote locations.

\section{Research methods}

\subsection{Data and Sample}

The analyses are based upon data from the last (i.e. the 6th) wave of the European Working Conditions Survey carried out in 2015 (EUROFOUND, 2017) in a random sample of European workers (employees and self-employed). This cross-sectional and single respondent-based survey provides a comprehensive picture of the characteristics of work processes in Europe as perceived by the workers themselves. Nonetheless, we selected this dataset as it includes several questions that can be used to operationalize the concepts included in our 
hypotheses, including an entirely renewed battery of items concerning a broad variety of work locations other than the conventional workplace that can be used to operationally distinguish between home-based and mobile e-workers. In addition, it provides representative data at European level, thus offering the great benefit of enhanced generalizability, helping to overcome the problem of law sample size faced by the majority of existing studies.

Our sample contains 1.380 home-based e-workers and 2.574 mobile e-workers. Workers of both groups have similar age within the age range 35 to 54 (58\% of home-based e-workers and $53 \%$ of mobile ones. They work as employees with a full-time contract (more than 80\%) and predominantly in the tertiary sector (around 66\%). Managers are only $12 \%$ in both groups. Now we look at their differences. Home-based e-workers are women $(54 \%)$ and they are mainly from UK (21\%), France (17\%), Germany (8\%), Spain (8\%) and Italy (2\%). They have a level of education higher than the bachelor's degree $(63 \%)$ and they work in large enterprises with more than 250 employees $(41 \%)$ as professionals (56\%). Mobile e-workers are men $(63 \%)$ and they are mainly from in UK (16\%), France (15\%), Germany (12\%), Spain (8\%) and Italy $(5 \%)$. They have a level of education higher than the bachelor's degree (42\%) and they work in medium-large businesses with 10 to 249 employees (40\%), as professionals $(28 \%)$ and technicians and associate professionals $(25 \%)$.

Table 1 shows that perception of discretion over different aspects of work is higher among home-based and mobile e-workers than among traditional workers (who do not frequently use digital technologies for work and work daily at the company's premises and less often or never in other locations). For example, 70\% of home-based e-workers and $68 \%$ of mobile ones report to have discretion over work pace against the $43 \%$ of traditional workers

However, both groups of remote e-workers suffer from greater work intensification than traditional workers, in particular with respect to frequent work interruptions (around $45 \% \mathrm{vs} 27 \%$ ) and the demand to work during free time (more than $30 \%$ vs. $13 \%$ ).

Note that within the remote e-workers group, home-based e-workers report to have autonomy over work goals $(63 \%)$ and discretion over work methods (82\%) more frequently than mobile e-workers (55\% and 64\%), but they also more often work during their free time (59\% vs. 30\%). On the other hands, mobile e-workers more frequently report to enjoy discretion over work schedule (50\% vs. $30 \%)$, but they are also subject to a greater number of time pressures ( $42 \%$ vs. $30 \%$ ).

Table 1. Relative frequencies of different dimensions of autonomy, discretion, and work intensification

\begin{tabular}{|c|c|c|c|}
\hline & $\begin{array}{l}\text { Traditional } \\
\text { workers }\end{array}$ & $\begin{array}{l}\text { Home-based } \\
\text { e-workers }\end{array}$ & $\begin{array}{l}\text { Mobile } \\
\text { e-workers }\end{array}$ \\
\hline Autonomy over work goals & $42 \%$ & $63 \%$ & $55 \%$ \\
\hline Discretion over work methods & $48 \%$ & $82 \%$ & $64 \%$ \\
\hline Discretion over work pace & $43 \%$ & $70 \%$ & $68 \%$ \\
\hline Autonomy in the choice of work colleagues & $25 \%$ & $35 \%$ & $38 \%$ \\
\hline Involvement in autonomous work groups & $54 \%$ & $61 \%$ & $66 \%$ \\
\hline Autonomy in the organizational choices of one's department/company & $43 \%$ & $63 \%$ & $61 \%$ \\
\hline Discretion over work schedule & $27 \%$ & $30 \%$ & $50 \%$ \\
\hline Working at high speed/to tight deadlines & $43 \%$ & $45 \%$ & $51 \%$ \\
\hline Time pressure & $38 \%$ & $30 \%$ & $42 \%$ \\
\hline Frequent work interruptions & $27 \%$ & $45 \%$ & $44 \%$ \\
\hline $\begin{array}{l}\text { Hours per day ( } \% \text { of workers that work between } 10 \text { and } 20 \text { times a month } \\
\text { more than } 10 \text { hours) }\end{array}$ & $4 \%$ & $6 \%$ & $8 \%$ \\
\hline $\begin{array}{l}\text { Hours per day ( } \% \text { of workers that does not work in a month more than } 10 \\
\text { hours per day) }\end{array}$ & $70 \%$ & $48 \%$ & $51 \%$ \\
\hline Working during free time & $13 \%$ & $59 \%$ & $30 \%$ \\
\hline Lack of recovery time & $18 \%$ & $32 \%$ & $29 \%$ \\
\hline
\end{tabular}

The sample descriptive data also suggest that the perceived level of occupational stress increases with the intensive use of digital technology and high mobility. The $75 \%$ of home-based e-workers and mobile e-workers experience stress at work at least sometimes compared to the $61 \%$ of traditional workers. Moreover, $31 \%$ of home-based e-workers and $36 \%$ of mobile ones report to experience stress most of the time or always compared to the $24 \%$ of traditional workers. 


\subsection{Measures}

Home-based e-workers are defined as individuals who always or almost always use mobile devices for work and work several times per month at home and never/less often in other remote locations.

Mobile e-workers include individuals who always or almost always use mobile devices for work and work several times per month in any location other than home and their main workplace and never/less often at home.

Occupational stress is measured by an item assessing how often the worker experiences stress during work ( $1=$ Never; $5=$ Always).

Involvement in autonomous work groups is measured by a binary item assessing whether the worker works in a team that has common tasks and plan its work.

Autonomy over work goals is measured by an item assessing how often the worker participates in goal setting (1= Never; $5=$ Always $)$.

Autonomy in the choice of work colleagues is measured by an item assessing how often the worker can choose his own colleagues $(1=$ Never; $5=$ Always $)$.

Autonomy in the organizational choices of one's department/company is measured by an item assessing how often the worker is involved in the choices regarding the organization of his department/company $((1=$ Never; $5=$ Always).

Discretion over work methods is the mean of the z-scores of four binary items assessing whether the worker can assess himself the quality of his work; solve unforeseen problems on his own; choose/change the order of tasks and the methods of work and a variable assessing how often the worker can apply his ideas into his work $(1=$ Never; $5=$ Always).

Discretion over work pace is the mean of the $\mathrm{z}$-scores of a dummy variable assessing if the worker can change/choose the speed/rate of work and a variable assessing how often he can take a break when he wishes $(1=$ Never; $5=$ Always).

Discretion over work schedule is measured by a dummy variable with $1=$ the worker can choose his work schedule within certain limits.

Working at very high speed/to tight deadlines is the mean of the z-scores of three items assessing if the worker's job involves working at high speed and to tight deadlines $(1=$ Never, $7=$ all of the time) and if the worker has enough time to do his job ( $1=$ Always; $5=$ Never $)$.

Time pressure is the mean of five dummy variables assessing whether the worker's pace of work depends on demands from customers, colleagues, bosses, performance or productivity targets, the speed of a machine/movement of a product.

Frequent work interruptions is measured by an item assessing how often the worker has to deal with work interruptions ( $1=$ Never, $4=$ Very often).

Long working hours is measured by a continuous variable assessing how many times a month the worker works more than 10 hours per day.

Working during free time is measured by an item assessing how often the individual works in his free time to meet work demands ( $(1=$ Never, $5=$ Daily $)$.

Lack of recovery time is measured by a binary item assessing if the worker has had less than 11 hours to recovery after work at least once in the last month.

We also consider demographic and employment variables (i.e. age, gender, education, employment contract, occupation, sector) and organizational variables (i.e. supervisor and co-workers' support, having undergone some forms of training, on the job learning, being subject to variable pay schemes, perceived skill match and task complexity) as controls.

\subsection{Analysis Technique}

This study computes univariate analysis of variance (ANOVA) to test whether the perceived level of occupational stress is statistically different among home-based and mobile e-workers compared to traditional workers. Moreover, it calculates multivariate analysis of variance (MANOVA) to test whether home-based and mobile e-workers differ from each other in terms of autonomy, discretion and work intensification variables as well as other organizational variables used as controls. Note that, the ANOVA for all the just mentioned 
work-related variables conduce to the same results.

Like prior studies (e.g. Suh \& Lee, 2017), to evaluate whether the direct relationships between autonomy, discretion and work intensification and their interaction effects on individual wellbeing vary depending on the mode of spatial flexibility, this paper estimates multiple OLS regressions separately for home-based and mobile e-workers. The econometric strategy compares nested specifications. Namely, every regression model includes different blocks of variables. Variables of autonomy and discretion, on the one hand, and of work intensification, on the other, define two distinct blocks, other organizational controls enter in another block and finally demographic and employment controls in another one. This analysis enables us to establish the relative contribution of each set of variables in terms of explained variance and to choose an optimal model. We report both unstandardized and standardized coefficients of the optimal model to explore the statistical significance of the coefficients and their relative importance to determine the level of perceived occupational stress. Moreover, we assess the variance inflation factor (VIF) of each regressor of the optimal model in order to check for multi-collinearity (Cohen, Cohen, West, \& Aiken, 2003). Finally, the optimal model is augmented by interactions to analyze how the level of occupational stress due to work intensification varies with the presence of autonomy or discretion or with increasing levels of autonomy or discretion. In other terms, we assess whether and to what extent autonomy and discretion variables moderate the negative influence of work intensification on perceived occupational stress. The interactions are estimated one autonomy and discretion variable (moderator) at a time. In the case of the moderating models, we only report unstandardized beta coefficients along with their standard errors, because standardized beta coefficients for interaction terms are not interpretable (Frazier, Tix, \& Baron, 2004). To conduct this statistical analysis, we use SPSS.

\section{Results}

The descriptive statistics suggest that the perceived level of occupational stress increase with the intensive use of digital technologies and high mobility: the $31 \%$ of home-based e-workers and the $36 \%$ of mobile ones report to experience stress at work most of the time or always compared to the $24 \%$ of traditional workers. The ANOVA test confirms this result showing that home-based and mobile e-workers statistically differ from traditional ones in terms of perceived work-related stress $(\mathrm{F}$ test $=162, \mathrm{p}<0.01$ ). The Tukey's post-hoc procedure, presented in Table 2, also reveals that all the groups are statistically different from each other at a level at least equal to $5 \%$.

Table 2. Multiple comparisons of different levels of perceived occupational stress among traditional workers, home-based e-workers and mobile e-workers

\begin{tabular}{lllllll}
\hline \multirow{2}{*}{ (I) Group } & \multirow{2}{*}{ Mean Difference (I-J) } & Std. Error & $\mathrm{p}$-value & \multicolumn{2}{c}{$95 \%$ Confidence Interval } \\
\cline { 6 - 7 } & & & & & Lower Bound & Upper Bound \\
\hline & 3 & $-0.32^{*}$ & 0.03 & 0.00 & -0.41 & -0.24 \\
& 1 & $0.42^{*}$ & 0.02 & 0.00 & -0.49 & -0.36 \\
& 3 & $-0.10^{*}$ & 0.03 & 0.00 & 0.24 & 0.41 \\
& 1 & $0.43^{*}$ & 0.04 & 0.04 & -0.20 & 0.00 \\
& 2 & $0.21^{*}$ & 0.02 & 0.00 & 0.36 & 0.49 \\
\end{tabular}

Notes. * indicates that the mean difference is significant at the 0.05 level. Group 1: Traditional workers, Group 2: Home-based e-workers. Group3: Mobile e-workers.

We now concentrate on home-based and mobile e-workers, i.e. the main focus of the present study. The descriptive statistics show that these two groups differ with respect to several dimensions of autonomy, discretion and work intensification (see Table 1). The MANOVA test confirms this, specifically it shows statistically significant differences in terms of the following blocks of variables: autonomy and discretion $(\mathrm{F}=$ 18.56, $\mathrm{p}<0.01)$, work intensification $(\mathrm{F}=79.04, \mathrm{p}<0.01)$ as well as organizational controls $(\mathrm{F}=5.82, \mathrm{p}<0.01)$. The MANOVA test that simultaneously consider all the three blocks above yields to the same conclusion $(\mathrm{F}=$ $5.82, \mathrm{p}<0.01)$.

To conclude, the MANOVA results suggest that the work-related variables that possibly contribute to the perceived levels of occupational stress are different across the two groups.

Therefore, to determine the relative contribution of the variables potentially related to occupational stress in each group, we estimate multiple OLS regressions considering separately home-based and mobile e-workers. Table 3 shows the optimal specification in terms of explained variance for both home-based e-workers (Adjusted 
$\mathrm{R}^{2}=0.1952$ ) and mobile ones (Adjusted $\mathrm{R}^{2}=0.1547$ ).

We use unstandardized and standardized coefficients of the optimal models to explore the statistical relevance of the estimated coefficients, their relative weight and to test the theoretical hypotheses outlined in the previous section. The variance inflation factor (VIF) of each regressor is less than 1.5 and less than 1.7 respectively suggesting no multi-collinearity problem in these regressions.

Table 3. Perceived occupational stress: final OLS regression models

\begin{tabular}{|c|c|c|c|c|}
\hline \multirow[b]{2}{*}{ Regressors } & \multicolumn{2}{|c|}{ Home-based e-workers } & \multicolumn{2}{|c|}{ Mobile e-workers } \\
\hline & $\begin{array}{l}\text { Unstandardized } \\
\text { coefficients }\end{array}$ & $\begin{array}{l}\text { Standardized } \\
\text { coefficients }\end{array}$ & $\begin{array}{l}\text { Unstandardized } \\
\text { coefficients }\end{array}$ & $\begin{array}{l}\text { Standardized } \\
\text { coefficients }\end{array}$ \\
\hline Autonomy over work goals & $\begin{array}{l}-0.057^{*} \\
(0.029)\end{array}$ & -.073 & $\begin{array}{l}-0.016 \\
(0.021)\end{array}$ & -.020 \\
\hline Discretion over work methods & $\begin{array}{l}0.112 \\
(0.087)\end{array}$ & .046 & $\begin{array}{l}0.006 \\
(0.058)\end{array}$ & .003 \\
\hline Discretion over work pace & $\begin{array}{l}-0.196 * * * \\
(0.051)\end{array}$ & -.140 & $\begin{array}{l}-0.187^{* * *} \\
(0.036)\end{array}$ & -.129 \\
\hline Autonomy in the choice of work colleagues & $\begin{array}{l}0.016 \\
(0.024)\end{array}$ & .022 & $\begin{array}{l}0.01 \\
(0.018)\end{array}$ & .014 \\
\hline Involvement in autonomous work groups & $\begin{array}{l}0.15^{* *} \\
(0.069)\end{array}$ & .066 & $\begin{array}{l}-0.055 \\
(0.053)\end{array}$ & -.023 \\
\hline $\begin{array}{l}\text { Autonomy in the organizational choices of one's } \\
\text { department/company }\end{array}$ & $\begin{array}{l}0.034 \\
(0.032)\end{array}$ & .041 & $\begin{array}{l}0.023 \\
(0.023)\end{array}$ & .027 \\
\hline Discretion over work schedule & $\begin{array}{l}-0.181^{* *} \\
(0.072)\end{array}$ & -.085 & $\begin{array}{l}-0.075 \\
(0.049)\end{array}$ & -.034 \\
\hline Working at high speed/to tight deadlines & $\begin{array}{l}0.214^{* * *} \\
(0.062)\end{array}$ & .113 & $\begin{array}{l}0.240^{* * *} \\
(0.042)\end{array}$ & .128 \\
\hline Time pressure & $\begin{array}{l}0.200 \\
(0.131)\end{array}$ & .047 & $\begin{array}{l}0.320^{* * *} \\
(0.095)\end{array}$ & .075 \\
\hline Frequent work interruptions & $\begin{array}{l}0.182 * * * \\
(0.036)\end{array}$ & .163 & $\begin{array}{l}0.195 * * * \\
(0.028)\end{array}$ & .165 \\
\hline Long working hours & $\begin{array}{l}-0.002 \\
(0.0025)\end{array}$ & -.024 & $\begin{array}{l}0.001 \\
(0.002)\end{array}$ & .013 \\
\hline Working during free time & $\begin{array}{l}0.150^{* * *} \\
(0.03)\end{array}$ & .167 & $\begin{array}{l}0.096^{* * *} \\
(0.024)\end{array}$ & .090 \\
\hline Lack of recovery time & $\begin{array}{l}0.114 \\
(0.070)\end{array}$ & .051 & $\begin{array}{l}0.148^{* * *} \\
(0.054)\end{array}$ & .059 \\
\hline Supervisor's support & $\begin{array}{l}-0.09 * * \\
(0.04)\end{array}$ & -.079 & $\begin{array}{l}-0.113 * * * \\
(0.031)\end{array}$ & -.095 \\
\hline Colleagues' support & $\begin{array}{l}-0.085 \\
(0.057)\end{array}$ & -.050 & $\begin{array}{l}-0.138^{* * * *} \\
(0.043)\end{array}$ & -.080 \\
\hline Complex tasks & $\begin{array}{l}0.260^{* * * *} \\
(0.087)\end{array}$ & .090 & $\begin{array}{l}0.208^{* * *} \\
(0.063)\end{array}$ & .076 \\
\hline On the job learning & $\begin{array}{l}-0.147 \\
(0.157)\end{array}$ & -.033 & $\begin{array}{l}-0.103 \\
(0.083)\end{array}$ & -.030 \\
\hline Skill match & $\begin{array}{l}-0.068 \\
(0.055)\end{array}$ & -.040 & $\begin{array}{l}-0.090^{* *} \\
(0.035)\end{array}$ & -.056 \\
\hline Training & $\begin{array}{l}-0.039 \\
(0.071)\end{array}$ & -.016 & $\begin{array}{l}-0.012 \\
(0.054)\end{array}$ & -.005 \\
\hline Variable pay & $\begin{array}{l}0.016 \\
(0.064)\end{array}$ & .007 & $\begin{array}{l}-0.061 \\
(0.047)\end{array}$ & -.027 \\
\hline Adjusted $\mathrm{R}^{2}$ & 0.1952 & 0.1952 & 0.1547 & 0.1547 \\
\hline F-test (p-value) & 0.24 & 0.24 & 0.55 & 0.55 \\
\hline
\end{tabular}

Notes. $* * * \mathrm{p} \leq 0.01 ; * * \mathrm{p} \leq 0.05 ; * \mathrm{p} \leq 0.1$. Robust standard errors in parenthesis. $\mathrm{F}$ test for the null that all single non- significant coefficients are also jointly not relevant. Demographic and employment controls are also included. VIF always $<1.5$ in the model regarding home-based e-workers and $<1.7$ in that concerning mobile ones.

Gender, education, age and other employment variables explain less than $1 \%$ of the variation in work-related stress perceived by home-based and mobile e-workers, for this reason we do not report the estimated coefficients 
for these controls. Regarding the organizational controls, they are statistically significant, the supervisor's support reduces the level of stress $(\beta=-0.079, \mathrm{p} \leq 0.05$ and $\beta=-0.095, \mathrm{p} \leq 0.01)$, while task complexity increases it for both groups $(\beta=0.09, p \leq 0.01$ and $\beta=0.076, p \leq 0.01)$. The colleagues' support and perceived skill match are also relevant to reduce the perception of occupational stress among mobile e-workers $(\beta=-0.08, p \leq 0.01$ and $\beta=-0.056, \mathrm{p} \leq 0.05)$.

Hypothesis 1 suggests that in the perception of remote e-workers, discretion and autonomy negatively relate to occupational stress. Table 3 shows that this hypothesis is supported for home-based e-workers for the following dimensions: autonomy over work goals $(\beta=-0.073, p \leq 0.10)$, discretion over work pace $(\beta=-0.140, p \leq 0.01)$, discretion over work schedule $(\beta=-0.085, \mathrm{p} \leq 0.05)$. On the contrary, discretion over work methods, autonomy in the choice of work colleagues and autonomy in the organizational choices of one's department/company are not statistically significant. Unexpectedly, taking part in autonomous work group significantly increases home-based e-workers' perception of work-related stress $(\beta=0.066, \mathrm{p} \leq 0.05)$. This finding aligns with previous research highlighting that working as part of an autonomous team requires repeated adjustments and modifications based on information and immediate reactions from others, as well as the exchange of tacit knowledge and complex ideas. This in turn asks for face-to-face interactions while making remote e-work and the related standardized communication unsuitable to support such dynamics (Boell et al., 2016). For mobile e-workers, Table 3 highlights that discretion over work pace is the only dimension which significantly reduces perceived work-related stress $(\beta=-0.129 \mathrm{p} \leq 0.01)$. Thus, for this group, Hypothesis 1 is only partially supported.

Hypothesis 2 posits that in the remote e-workers' perception, work intensification positively relates to occupational stress. As shown in Tables 3 this hypothesis is accepted for both groups. More specifically, working during one's free time $(\beta=0.167, p \leq 0.01)$, frequent work interruptions $(\beta=0.163, p \leq 0.01)$, and working at high speed and to tight deadlines $(B=0.113, p \leq 0.01)$ are statistically significant determinants of home-based e-workers' perceived occupational stress. The adverse effects of work intensification appear even more pronounced among mobile e-workers. In fact, together with frequent work interruptions $(\beta=0.165, p \leq 0.01)$, working at high speed and to tight deadlines $(\beta=0.128, \mathrm{p} \leq 0.01)$, and working in one's free time $(\beta=0.09$, p $\leq 0.01)$, time pressure $(\beta=0.075, \mathrm{p} \leq 0.01)$ and lack of recovery time $(\beta=0.059, \mathrm{p} \leq 0.01)$ also significantly increase the perception of occupational stress in this group. Hypothesis 3a states that discretion and autonomy moderate the association between work intensification and occupational stress as perceived by remote e-workers. Results are reported in Table 4 (relating to home-based e-workers) and 5 (relating to mobile ones), which only present the statistically significant interactions.

Table 4 indicates that discretion over work pace $(\beta=-0.118, p \leq 0.10)$ and discretion over work methods $(\beta=-$ $0.365, p \leq 0.01$ ) reduce the stressful influence of working at high speed and to tight deadlines among home-based e-workers. Discretion over work methods also buffers the stressful effect of time pressure $(\beta=-0.536 p \leq 0.10)$. Moreover, autonomy over work goals and in the organizational choices of one's department/company alleviate the negative consequences of lack of recovery time $(\beta=-0.118, p \leq 0.01$ and $\beta=-0.144, p \leq 0.05)$. Therefore, Hypothesis $3 \mathrm{a}$ is supported for home-based e-workers: both autonomy and discretion have a moderating effect among home-based e-workers, and namely a buffering effect, i.e. the higher the perception of autonomy and discretion, the lower the perception of the stressful effects of work intensification.

For mobile e-workers, Table 5 shows that discretion over work pace intensifies the negative effect of working at high speed and to tight deadline $(\beta=0.117, \mathrm{p} \leq 0.05)$ increasing its magnitude by around $50 \%(\beta=0.223+0.117)$. In addition discretion over work schedule worsens the impact of both frequent work interruptions and working in one's free time on mobile e-workers' perceived occupational stress $(\beta=0.135, \mathrm{p} \leq 0.01, \beta=0.078, \mathrm{p} \leq 0.10)$. In both cases, the impact of work intensification is double under conditions of high discretion over work schedule $(\beta=0.122+0.135, \beta=0.053+0.078)$. Contrarily, no autonomy variable has statistically significant moderating effects. Thus, for mobile e-workers, Hypothesis $3 \mathrm{a}$, which states that both autonomy and discretion have moderating effects, is only partially supported. Only discretion has a moderating effect among this group, and namely a boosting effect: the higher the perception of discretion, the higher the perception of the stressful effects of work intensification.

The results also highlight that the moderating role of discretion differs across the two groups of remote e-workers: for home-based e-workers, discretion alleviates the stressful implications of work intensification while for mobile e-workers, it intensifies such an adverse effect. Thus, Hypothesis $3 b$, which suggests that the moderating role of both autonomy and discretion differ depending on whether remote e-workers work at home or in not-fixed, remote locations, is partially supported. 
Table 4. Statistically significant moderating effects of autonomy and discretion among home-based e-workers

\begin{tabular}{|c|c|c|c|c|c|}
\hline & 1 & 2 & 3 & 4 & 5 \\
\hline \multicolumn{6}{|l|}{ Interactions } \\
\hline \multirow[t]{2}{*}{ Working at high speed/to tight deadlines } & $0.238 * * *$ & & & & \\
\hline & $(0.058)$ & & & & \\
\hline \multirow[t]{2}{*}{ Discretion over work pace } & $-0.222 * * *$ & & & & \\
\hline & $(0.049)$ & & & & \\
\hline \multirow[t]{2}{*}{ Working at high speed/to tight deadlines $\times$ discretion over work pace } & $-0.118 *$ & & & & \\
\hline & $(0.072)$ & & & & \\
\hline \multirow[t]{2}{*}{ Lack of recovery time } & & $0.524 * * *$ & & & \\
\hline & & $(0.182)$ & & & \\
\hline \multirow[t]{2}{*}{ Autonomy over work goals } & & -0.011 & & & \\
\hline & & $(0.029)$ & & & \\
\hline \multirow[t]{2}{*}{ Lack of recovery time $\times$ Autonomy over work goals } & & $-0.118 * * *$ & & & \\
\hline & & $(0.044)$ & & & \\
\hline \multirow[t]{2}{*}{ Lack of recovery time } & & & $0.649 * * *$ & & \\
\hline & & & $(0.210)$ & & \\
\hline \multirow[t]{2}{*}{ Autonomy in the organizational choices of one's department/company } & & & $0.078 * * *$ & & \\
\hline & & & $(0.037)$ & & \\
\hline \multirow{2}{*}{$\begin{array}{l}\text { Lack of recovery time } \times \text { Autonomy in the organizational choices of one's } \\
\text { department/company }\end{array}$} & & & $-0.144 * *$ & & \\
\hline & & & $(0.056)$ & & \\
\hline \multirow[t]{2}{*}{ Working at high speed/to tight deadlines } & & & & $0.321 * * *$ & \\
\hline & & & & $(0.068)$ & \\
\hline \multirow[t]{2}{*}{ Discretion over work methods } & & & & 0.061 & \\
\hline & & & & $(0.080)$ & \\
\hline \multirow[t]{2}{*}{ Working at high speed/to tight deadlines $\times$ discretion over work methods } & & & & $-0.365^{* * *}$ & \\
\hline & & & & $(0.128)$ & \\
\hline \multirow[t]{2}{*}{ Time pressure } & & & & & $0.416^{* *}$ \\
\hline & & & & & $(0.162)$ \\
\hline \multirow[t]{2}{*}{ Discretion over work methods } & & & & & $0.262 *$ \\
\hline & & & & & $(0.138)$ \\
\hline \multirow[t]{2}{*}{ Time pressure $\times$ discretion over work methods } & & & & & $-0.536^{*}$ \\
\hline & & & & & $(0.305)$ \\
\hline Adjusted $R^{2}$ final model + interaction term & 0.1966 & 0.1998 & 0.2035 & 0.2018 & 0.1970 \\
\hline
\end{tabular}

Notes. ${ }^{* *} \mathrm{p} \leq 0.01 ; * * \mathrm{p} \leq 0.05 ; * \mathrm{p} \leq 0.1$. Unstandardized coefficients. Robust standard errors in parenthesis.

Table 5. Statistically significant moderating effects of autonomy and discretion among mobile e-workers

\begin{tabular}{|c|c|c|c|}
\hline & 1 & 2 & 3 \\
\hline \multicolumn{4}{|l|}{ Interactions } \\
\hline \multirow[t]{2}{*}{ Working at very high speed/to tight deadlines } & $0.223 * * *$ & & \\
\hline & $(0.041)$ & & \\
\hline \multirow[t]{2}{*}{ Discretion over work pace } & $-0.202 * * *$ & & \\
\hline & $(0.036)$ & & \\
\hline \multirow[t]{2}{*}{ Working at high speed/to tight deadlines $\times$ Discretion over work pace } & $0.117 * *$ & & \\
\hline & $(0.051)$ & & \\
\hline \multirow[t]{2}{*}{ Frequent work interruptions } & & $0.122 * * *$ & \\
\hline & & $(0.038)$ & \\
\hline \multirow[t]{2}{*}{ Discretion over work schedule } & & 0.016 & \\
\hline & & $(0.022)$ & \\
\hline \multirow[t]{2}{*}{ Frequent work interruptions $\times$ discretion over work schedule } & & $0.135^{* * *}$ & \\
\hline & & $(0.051)$ & \\
\hline \multirow[t]{2}{*}{ Working during free time } & & & $0.053^{*}$ \\
\hline & & & $(0.031)$ \\
\hline \multirow[t]{2}{*}{ Discretion over work schedule } & & & $-0.230 * *$ \\
\hline & & & $(0.104)$ \\
\hline \multirow[t]{2}{*}{ Working during free time $\times$ Discretion over work schedule } & & & $0.078^{*}$ \\
\hline & & & $(0.044)$ \\
\hline
\end{tabular}




$\begin{array}{llll}\text { Adjusted } R^{2} & \text { final model }+ \text { interaction term } & 0.1568 & 0.1574\end{array}$

Notes. $* * * \mathrm{p} \leq 0.01 ; * * \mathrm{p} \leq 0.05 ; * \mathrm{p} \leq 0.1$. Unstandardized coefficients. Robust standard errors in parenthesis.

\section{Discussion and Implications}

This study contributes to the debate on the micro-level consequences of novel ways of organizing work enabled by digitalization, providing a quantitative, more nuanced understanding of the implications of remote e-work on individual wellbeing, namely on occupational stress. Overall, this research makes two contributions.

First, it provides new empirical evidence on the mode of spatial flexibility as a key variable to unpack the stressful effects of remote e-work, by highlighting that the negative consequences of this work mode on occupational stress are stronger for mobile e-workers. We find that mobile e-workers experience higher levels of occupational stress compared to home-based ones and perceive a greater number of work intensification dimensions as drivers of occupational stress. Home-based e-workers perceive that autonomy (namely over work goals) directly decreases occupational stress and buffers work intensification (i.e. autonomy over work goals and in the organizational choices of their department/company), while mobile e-workers do not perceive any positive influence of autonomy on occupational stress. One possible explanation of this is that making autonomous decisions about one or more aspects of work asks for an ongoing dynamic exchange of complex ideas and tacit knowledge with superiors and colleagues that typically requires face-to-face interactions between individuals co-located in the same physical space. Previous research suggests that, unlike home-based e-workers, mobile ones hardly have the opportunity to commute to the main workplace to communicate with superiors and colleagues face-to-face. Since they mainly work from geographically dispersed settings distant from the company's premises, they have to rely more on new digital technologies-mediated interactions that however hinder coordination with their superiors and colleagues, hampering the effective exercise of autonomy (Boell et al., 2016; Neirotti et al., 2013; Vartiainen \& Hyrkkänen, 2010). Finally, our study shows that mobile e-workers are less likely to perceive the beneficial effects of discretion. As compared to home-based e-workers, mobile ones perceive that only the possibilities to choose when to work significantly reduces occupational stress. Moreover, while for home-based e-workers discretion (i.e. over work pace and work methods) offsets the stressful implications of work intensification, for mobile e-workers it (i.e. discretion over work pace and work schedule) intensifies such adverse effects.

Second, our research underscores the importance of differentiating not only between home-based and mobile e-workers, but also between autonomy and discretion as a way to advance our understanding of the so-called 'autonomy paradox'. Specifically, our results show that what is likely to boost the stressful impact of work intensification is the possibilities to choose when to work, that is a dimension relating to discretion rather than to autonomy. In line with Thompson (1967), we contend that discretion is primarily an organizational requirement, typical of highly uncertain work situations; far from being a paradox, its exercise may negatively affect individual wellbeing. We provide further evidence of this, showing that in the context of remote e-work discretion is more likely to boost the stressful impact of work intensification when work is mobile, ubiquitous, demanding to managing complex relationships with a high number of different interest groups and thus more uncertain. At the same time, we do not find that autonomy increases work intensification, neither among mobile e-workers, nor among home-based e-workers (for whom it buffers the adverse impact of work intensification). In summary, this study does not confirm the existence of an autonomy paradox associated with remote e-work. Contrarily, it suggests that such a paradox is more likely to surface when research is based on the JD-C and JD-R frameworks or other approaches that like the former ambiguously define autonomy in terms of what should be more properly conceptualized as discretion.

Regarding managerial implications, our research questions the EUROFOUND and ILO's (2017) recommendation to afford greater discretion to individuals in order to manage the negative effects of remote e-work, as this may actually intensify the problem for mobile e-workers. Our results also suggest that the prevention approach targeting "the right to disconnect from work", which collective agreements, governmental action and legislative initiatives have recently promoted in some European countries, may be useless for mobile e-workers. For these, the main conditions of stress are, in fact, frequent work interruptions, high work speed and tight deadlines occurring within their normal working day. Rather than a blanket approach, we thus recommend corporate leaders to develop appropriate strategies to fit the needs of different groups of remote e-workers. Measures ensuring the disconnection from work can be an appropriate response for home-based e-workers who suffer most from having to work in free time to meet work demands. They are also the most affected by the stressful consequences of being members of an autonomous work group; to contrast this phenomenon, organizations could provide them with greater opportunities for co-location in the same physical space with their bosses and colleagues as this facilitates the sharing of complex ideas and tacit knowledge nurturing the effective 
exercise of autonomy. To address the major conditions of stress of mobile e-work, instead, organizations need to develop much more creative alternatives, such as developing practices to tailor mobile e-work to fit workers and not only the organizational needs (Putnam et al., 2014). This calls for the adoption of a new perspective, which conceives the coordination of individuals' tasks and their interdependences with others as a negotiating process between the organization, the employee and outside stakeholders (i.e. clients). Such approach is in line with the principles laid down by the national and European regulations on health and safety at work, which force organizations to prioritize the primary prevention of stress conditions rather than the protection of workers already exposed to those conditions by such measures as rest pauses, limits to the number of working hours and disconnection from work. Primary prevention relies on a comprehensive analysis and redesign of work in which workers take an active part. Today, such analysis may benefit from the new opportunities to collect and analyze data on work processes enabled by the same new digital technologies that workers use to perform work from remote locations. This in turn requires an unbiased reflection on how to strike a balance between the workers right to privacy as well as to health.

\section{Acknowledgments}

The authors gratefully acknowledge financial support from the grant FAR2019 (awarded by the University of Modena and Reggio Emilia, Italy).

\section{References}

Albano, R., Curzi, Y., Parisi, T., \& Tirabeni, L. (2018). Perceived autonomy and discretion of mobile workers. Studi Organizzativi, 2, 31-61. https://doi.org/10.3280/SO2018-002002

Allen, T. D., Golden, T. D., \& Shockley, K. M. (2015). How effective is telecommuting? Assessing the status of our scientific findings. Psychological Science in the Public Interest, 16, 40-68. https://doi.org/10.1177/1529100615593273

Azad, B., Salamoun, R., Greenhill, A., \& Wood-Harper, T. (2016). Performing projects with constant connectivity: interplay of consulting project work practices and smartphone affordances. New Technology, Work and Employment, 31(1), 4-25. https://doi.org/10.1111/ntwe.12061

Biron, M., \& Van Veldhoven, M. (2016). When control becomes a liability rather than an asset: Comparing home and office days among part-time teleworkers. Journal of Organizational Behavior, 37, 1317-1337. https://doi.org/10.1002/job.2106

Boell, S. K., Cecez-Kecmanovic, D., \& Campbell, J. (2016). Telework paradoxes and practices: the importance of the nature of work. New Technology, Work and Employment, 31(2), 114-131. https://doi.org/10.1111/ntwe.12063

Cavazotte, F., Lemos, A. H., \& Villadsen K. (2014). Corporate smart phones: professionals' conscious engagement in escalating work connectivity. New Technology, Work and Employment, 29, 72-87. https://doi.org/10.1111/ntwe.12022

Charalampous, M., Grant, C. A., Tramontano, C., \& Michailidis, E. (2018). Systematically reviewing remote e-workers' well-being at work: a multidimensional approach. European Journal of Work and Organizational Psychology. https://10.1080/1359432X.2018.1541886.

Cohen, J., Cohen, P., West, S. G., \& Aiken, L. S. (2003). Applied multiple regression/correlation analysis for the behavioral sciences (3rd ed.). Mahwah, NJ: Lawrence Erlbaum Associates.

Demerouti, E., Bakker, A. B., Nachreiner, F., \& Schaufeli, W. B. (2001). The job demands-resources model of burnout. Journal of Applied Psychology, 86, 499-512. https:// 10.1037/0021-9010.86.3.499 .

Eurofound, \& the International Labour Office. (2017). Working anytime, anywhere: The effects on the world of work. Luxembourg: Publications Office of the European Union, Geneva: the International Labour Office.

European Foundation for the Improvement of Living and Working Conditions (2017). European working conditions survey, 2015 (3rd ed.). Colchester, Essex: UK Data Archive. SN: 8098. http://dx.doi.org/10.5255/UKDA-SN-8098-3

Felstead, A., \& Henseke, G. (2017). Assessing the growth of remote working and its consequences for effort, well-being and work-life balance. New Technology, Work and Employment, 32, 195-212. https://doi.org/10.1111/ntwe.12097

Frazier, P. A., Tix, A. P., \& Baron, K. E. (2004). Testing moderator and mediator effects in counseling psychology research. Journal of Counseling Psychology, 51, 115-134. https://doi.org/10.1037/0022-0167.51.1.115

Gajendran, R. S., \& Harrison, D. A. (2007). The good, the bad, and the unknown about telecommuting: meta-analysis of psychological mediators and individual consequences. Journal of Applied Psychology, 
92(6), 1524-1541. https:// doi.org/10.1037/0021-9010.92.6.1524

Gajendran, R. S., Harrison, D. A., \& Delaney-Klinger, K. (2015). Are telecommuters remotely good citizens? Unpacking telecommuting's effects on performance via i-deals and job resources. Personnel Psychology, 68, 353-393. https://doi.org/10.1111/peps.12082

Garrett, R. K., \& Danziger, J. N. (2007). Which telework? Defining and testing a taxonomy of technology-mediated work at a distance. Social Science Computer Review, 25, 27-47. https://10.1177/0894439306293819

Golden, T. D., \& Veiga, J. F. (2005). The impact of extent of telecommuting on job satisfaction: resolving inconsistent findings. Journal of Management, 31, 301-318. https://doi.org/10.1177/0149206304271768

Grant, C. A., Wallace, L. M., \& Spurgeon, P. C. (2013). An exploration of the psychological factors affecting remote e-worker's job effectiveness, well-being and work-life balance. Employee Relations, 35, 527-546. https://doi.org/10.1108/ER-08-2012-0059

Karasek, R. (1979). Job demands, job decision latitude, and mental strain: Implications for job redesign. Administrative Science Quarterly, 24, 285-308. https://doi.org /10.2307/2392498

Kelliher, C., \& Anderson, D. (2010). Doing more with less? Flexible working practices and the intensification of work. Human Relations, 63, 83-106. https://doi.org/10.1177/0018726709349199

Konradt, U., Hertel, G., \& Schmook, R. (2003). Quality of management by objectives, task-related stressors, and non-task-related stressors as predictors of stress and job satisfaction among teleworkers. European Journal of Work and Organizational Psychology, 12, 61-79. https://doi.org/10.1080/13594320344000020

Maggi, B. (2016). De l'agir organisationnel. Un point de vue sur le travail, le bien-être, l'apprentissage (2nd ed.). Bologna: Tao Digital Library. https://doi.org/10.6092/unibo/amsacta/5439 (2003, 1st ed., Toulouse: Octarès Editions).

Mazmanian, M., Orlikowski, W. J., \& Yates, J. (2013). The autonomy paradox. The implications of mobile devices for knowledge professionals. Organization Science, 24, 1337-1357. https://doi.org/10.1287/orsc.1120.0806

Neirotti, P., Paolucci, E., \& Raguseo, E. (2013). Mapping the antecedents of telework diffusion: firm-level evidence from Italy. New Technology, Work and Employment, 28, 16-36. https://doi.org/10.1111/ntwe.12001

Putnam, L. L., Myers, K. K., \& Gailliard, B. M. (2014). Examining the tensions in workplace flexibility and exploring options for new directions. Human Relations, 67, 413-440. https://doi.org/10.1177/0018726713495704

Sardeshmukh, S. R., Sharma, D., \& Golden, T. D. (2012). Impact of telework on exhaustion and job engagement: a job demands and job resources model. New Technology, Work and Employment, 27, 193-207. https://doi.org/10.1111/j.1468-005X.2012.00284.x

Suh, A., \& Lee, J. (2017). Understanding teleworkers' technostress and its influence on job satisfaction. Internet Research, 27, 140-159. https:// doi.org/10.1108/IntR-06-2015-0181

ter Hoeven, C. L., \& Van Zoonen, W. (2015). Flexible work designs and employee well-being: examining the effects of resources and demands. New Technology, Work and Employment, 30, 237-255. https://doi.org/10.1111/ntwe.12052

Thompson, J. D (1967). Organizations in Action. New York: McGraw-Hill (2003, New Brunswick/London: Transaction Publishers).

Van Steenbergen, E. F., Van der Ven, C., Peeters, M. C. W., \& Taris, T. W. (2018). Transitioning towards new ways of working: do job demands, job resources, burnout, and engagement change? Psychological Reports, 121(4), 736-766. https://doi.org/10.1177/0033294117740134

Vartiainen, M., \& Hyrkkänen U. (2010). Changing requirements and mental workload factors in mobile multi-locational work. New Technology, Work and Employment 25(2), 117-135. https://doi.org/10.1111/j.1468-005X.2010.00243.x

Vendramin, P. (2007). Les métiers des TIC: un nomadisme coopératif. In A. F. Saint Laurent-Kogan, \& J. L. Metzger (Eds.), Où va le travail à l'ère du numérique? (pp. 89-104). Paris: Presses Des Mines. https://doi.org/10.4000/books.pressesmines. 1387

\section{Copyrights}

Copyright for this article is retained by the author(s), with first publication rights granted to the journal.

This is an open-access article distributed under the terms and conditions of the Creative Commons Attribution license (http://creativecommons.org/licenses/by/4.0/). 